\title{
The dimensional analysis of multidimensional stimuli by children
}

\author{
CHARLES C. SPIKER and JOAN H. CANTOR \\ University of Iowa, Iowa City, Iowa
}

\begin{abstract}
It was proposed that the accessibility of the dimensional structure of multidimensional stimuli can be assessed by observing the response latencies of children asked to name the dimensional values of multidimensional stimuli. One implication of this proposition is that response times (RTs) to partitioned stimuli, for which the dimensional values are spatially separated, should be faster than RTs to unitary stimuli. Another implication is that the difference between the RTs to unitary and partitioned stimuli should decrease with chronological age. Kindergarten, first-grade, and second-grade children were asked to name the dimensional values of partitioned and unitary multidimensional stimuli, and their RTs were recorded. It was found that the RTs to the partitioned stimuli were significantly faster than those to the unitary stimuli and that the difference in RTs was smaller for the second-grade children than for the younger children. It was concluded that the RT technique provides a valid index of the accessibility of the dimensional information.
\end{abstract}

For the past couple of decades, there has been considerable research on stimulus factors that affect the ease with which subjects can analyze multidimensional stimuli into dimensions. The concern began with research on adult human subjects (e.g., Garner, 1970; Lockhead, 1966; Shepard, 1964) and was subsequently extended into the developmental field (e.g., Kemler, 1982; Kemler \& Smith, 1979; Shepp \& Swartz, 1976; Smith, 1979). Research with both adults and children has led to a distinction between what Garner (1974) called "integral" and "separable" stimulus dimensions and Shepard (1964) referred to as "nonanalyzable" and "analyzable" stimulus dimensions. Several different tasks have been used to identify separable and integral dimensions; indeed, one of these tasks can be used to provide definitions of the concepts.

The brightness and saturation of colors are frequently offered as an example of a pair of integral dimensions for either adults or children. For adults, the brightness and size of stimuli are generally considered to be separable. In order to explicate the concepts of integrality and separability, it is necessary to describe some of the tasks that have been used in studying integral and sepa-

The authors wish to thank Ann Aschbacher and Janet Lyness for their excellence in collecting and tabulating the data. We are also deeply indebted to William J. Bach, James Barger, and Charles Swaney of the College Community School District in Cedar Rapids, Iowa, and to David L. Cronin, Richard E. Taylor, James D. Blank, and Donald J. Tvedte of the Iowa City School District for their outstanding cooperation. Funds for the purchase of computer time were made available by the Graduate College of the University of Iowa. Requests for reprints should be addressed to Charles C. Spiker, Department of Psychology, University of Iowa, Iowa City, Iowa 52242. rable dimensions. We will begin by describing three sets of stimuli that could be used in one of these tasks. One set consists of hues varying in brightness and saturation, with these two dimensions orthogonal to each other (the orthogonal set). A second set consists of hues varying in brightness and saturation, but with the two dimensions arranged in a correlated manner (the correlated set). The third set consists of hues varying in brightness (or saturation) only, with saturation (or brightness) held constant (the unidimensional set). When adult subjects are requested to classify these three sets of stimuli on the basis of a single dimension and under time pressure (the speeded classification test), it is found consistently that the correlated set is sorted faster than the unidimensional set, which in turn is sorted faster than the orthogonal set. When parallel sets, constructed from the size and brightness dimensions, are administered to comparable subjects, no differences are found in performance on the three sets. (See Kemler, 1983, and Shepp, 1983, for excellent discussions and summaries of this research.)

The two types of stimuli produce different results in another kind of task, referred to as the restricted classification task (Shepp, 1983). In this task, there are three stimuli, varying on two dimensions, presented on each trial. Two of them, say A and B, share the same value on one dimension, but differ markedly on the other. The third stimulus, C, differs from A and B in its values on both dimensions, but it is similar to B on both dimensions and differs considerably from $\mathrm{A}$ on one dimension. When subjects are asked on each trial to make the best classification, they may place together either the two that have the greatest overall similarity (i.e., B and C) or the two that share a value on one dimension (i.e., $\mathbf{A}$ and B). In other words, they may sort on the basis of 
overall similarity or on the basis of the dimensional structure of the stimuli.

In the restricted classification task, adults tend to classify stimuli constructed from dimensions identified as integral by the speeded classification task on the basis of overall similarity. Stimuli constructed from separable dimensions, however, are classified on the basis of dimensions (Kemler, 1983; Shepp, 1983).

Research with children suggests that a dichotomous division of dimensions into separable and integral may not be warranted. Rather, it appears that there may be, at least for children, a continuity between the two types of dimensions, with the dimensional structure readily accessible in some instances but, in other cases, accessible only with considerable difficulty or not at all. There are at least some dimensions that are separable for adults but that children find difficult to analyze into dimensional components. Thus, Smith and Kemler (1977) found that kindergarten children who had classified stimuli in the restricted classification task on the basis of overall similarity nevertheless used the names of dimensional values in their attempts to justify the basis of their classifications. The picture that emerges is one in which younger (e.g., kindergarten) children prefer to make overall similarity judgments, but can nevertheless use a good deal of dimensional information when necessary.

The investigation of the integral-separable continuum would be much facilitated by a convenient method of determining for the individual child the degree of accessibility of the dimensional information for any arbitrarily chosen separable dimensions. One possible method is to have children name the dimensional values of multidimensional stimuli and measure the latencies to respond. This possibility receives some support from Smith and Kemler (1977), who found that many kindergarten children were able to explain their classification choices in terms of dimensional values. The authors interpreted the availability of the names of dimensional values as evidence for the accessibility of the dimensional structure of the stimuli. The readiness with which individual children can do this should be reflected in their latencies to respond to requests to name the dimensional values of multidimensional stimuli.

The most direct test of the validity of the latency measure might appear to be a comparison of naming latencies for integral and separable stimuli. Unfortunately, it is not reasonable to ask adults, let alone children, to give the names of different brightnesses or saturation values. There is, however, another alternative. It has been demonstrated that if stimuli are constructed from integral dimensions by spatially separating the dimensional values, stimuli that adults respond to as if the dimensions were separable are produced (Garner \& Felfoldy, 1970; Shepp, 1983, p. 52). Consistent with this finding is our own experience that kindergarten and first-grade children use dimensional values as hypotheses more effectively if the dimensional values are spatially separated (Cantor \& Spiker, 1978; Spiker \& Cantor, 1983).

The present experiment was directed toward testing some implications of the hypothesis that the latencies of children's responses in naming the dimensional values reflect the degree of accessibility of the dimensional structure. Kindergarten, first-grade, and second-grade children were asked to name the dimensional values of multidimensional stimuli varying in hue, form, and size. Half of the stimuli were partitioned stimuli, constructed by spatially separating the different dimensional values, and the other half consisted of unitary stimuli constructed from the same dimensions. The time required to complete the naming of the dimensional values was recorded. It was predicted that the response times (RTs) would be faster to the partitioned stimuli than to the unitary stimuli, at least for the younger children, because the dimensional structure of the partitioned stimuli would be more readily accessible than that of the unitary stimuli. For the older children, the advantage of the partitioned stimuli was expected to be considerably reduced, if not eliminated altogether, since older children were expected to find the dimensional structure of unitary stimuli readily accessible. The RTs for both types of stimuli, of course, were expected to decline with increases in chronological age.

\section{METHOD}

\section{Subjects}

The subjects were 20 kindergarten, 20 first-grade, and 20 second-grade children from the Iowa City Community School District, who participated on a voluntary basis after their parents had returned consent slips. The mean chronological age (CA) for the kindergarten children was 74.5 months, with a range of 68 to 82 months. For the first-grade children, the mean CA was 85.3 months, with a range of 77 to 90 months. The mean CA for the second-graders was 96.4 months, with a range of 87 to 105 months. The data for 1 additional first-grade child were discarded because of an experimenter error. The data for 4 additional children, 2 kindergarteners and 2 first-graders, were discarded because the children could not consistently name the values on the stimulus dimensions.

\section{Stimulus Materials}

Two types of stimuli were used: one consisting of the unitary multidimensional stimuli and the other consisting of the partitioned multidimensional stimuli. The eight unitary stimuli consisted of all combinations of two sizes (large or small), two colors (red or blue), and two forms (square or circle). The dimensional values for each stimulus (e.g., large red square) were combined in a unitary figure cut out of colored cardboard and attached to the center of a white card, $6 \mathrm{~cm}$ square. The sides of the squares were $24 \mathrm{~mm}$ (large) and $14 \mathrm{~mm}$ (small), and the diameters of the circles were $25 \mathrm{~mm}$ (large) and $16 \mathrm{~mm}$ (small).

The same dimensional values constituting the unitary stimuli were used to prepare the partitioned stimuli. In this case, the three dimensional values for a given stimulus were spatially separated on the card, with a black line drawing of the appropriate form in the lower left corner, an irregularly shaped color patch in the lower right corner, and a vertical arrowhead representing size in the top center of the card. The forms had the same dimensions as the large stimuli previously described. The arrowhead was either $26 \mathrm{~mm}$ long (large) or $10 \mathrm{~mm}$ long (small). 


\section{Apparatus}

The apparatus consisted of a black wooden platform, $56 \mathrm{~cm}$ wide $\times 44 \mathrm{~cm}$ deep, which was bisected by a vertical partition $32 \mathrm{~cm}$ high. The apparatus was placed on a low table directly in front of the child, and the stimuli were presented on the front half of the platform, which was covered with white felt material. The stimulus materials not in use were placed out of the child's sight behind the partition.

\section{Procedure}

Each child was brought individually to a mobile research trailer located next to the school building and was seated in front of the apparatus. On each trial, four stimulus cards were placed on the table before the subject. The four cards presented on each trial were chosen such that each value on each dimension was represented twice. The child was asked to name all three dimensional values on each card, beginning with the value on the left. The children were encouraged to name the values in the order "size, color, form," although other orders were not recorded as incorrect. Sixteen such trials were administered, the first eight as practice trials and the last eight as the criterion task.

On half the trials for each child, all four cards contained partitioned stimuli, and on the other half, they contained unitary stimuli. Partitioned and unitary trials were alternated in the $A B B A B A A B$ fashion in both the first eight and the second eight trials, with half the children in each age group beginning with a partitioned-stimulus trial and the other half beginning with a unitary-stimulus trial.

After the child was seated at the apparatus, the experimenter informed the child that they were going to play a game with cards. The experimenter said, "I'd like you to go through and tell me what's on the cards. Start on this side (pointing to the child's left) and give the size first, then the color, and then the shape, like this." The experimenter then pointed to the first card and said, for example, "Big red square," and continued through the remaining three cards. The child was then asked to try it. Any mistakes made by the child were corrected when they were made. After the child correctly named the dimensional values, the experimenter said, "From now on, do it as quickly as you can without making mistakes."

The data sheet contained a coded description of all the stimuli for each trial, as well as a blank in which the time for each trial could be recorded. The experimenter started a stop watch when the child began reporting the dimensional values and stopped the watch when the last value had been named. Thus, the recorded time was the time it took the child to give the names for all 12 dimensional values on a given trial. In addition, the experimenter recorded on the coded description any dimensional value that the child failed to name properly or omitted. The order of naming the dimensional values for a given stimulus was neither recorded nor considered in designating errors.

The data collected from each child, therefore, included the time required to name the dimensional values and the number of errors for each of the eight trials of the criterion task.

\section{RESULTS}

The mean RTs (in seconds) and mean errors are shown in Table 1 for all three age groups under both stimulus conditions. It is apparent from Table 1 that RTs decrease from the kindergarten group to the secondgraders and that average RTs to the unitary stimuli are longer than those to the partitioned stimuli. The same trends appear in the error means of Table 1.

The RTs were subjected to an analysis of variance with grade as a between-subjects factor and stimuli as a within-subject factor. The main effects of grade $[F(2,57)=15.11, p<.001]$ and stimuli $[F(1,57)=$ $33.31, \mathrm{p}<.001]$ were significant. The grade $\mathrm{x}$ stimuli interaction was also significant $[F(2,57)=3.20, p<.05]$.

Follow-up tests on the main effect of grade indicated that the difference between the kindergarten mean and the first-grade mean was significant $[F(1,57)=5.00$, $p=.029]$, and the combined kindergarten and first-grade mean differed significantly from the second-grade mean $[F(1,57)=25.22, p<.001]$. The significant grade $x$ stimuli interaction reflects the fact that the difference between the partitioned-stimulus mean and the unitarystimulus mean was smaller for the second-grade children than for the younger groups.

The trends in the error data were similar to those in the RT data. Analysis of variance of the errors yielded significant statistics for the main effects, although at somewhat less impressive levels of significance. The grade $\mathrm{x}$ stimuli interaction showed the same pattern as that for RTs, as can be seen in Table 1, but was not statistically significant $[F(2,57)=1.78, p>.10]$.

\section{DISCUSSION}

For the most part, the results are consistent with the hypothesis that RTs reflect the ease of accessing the dimensional structure of stimuli. As expected, the younger children found the unitary-stimulus dimensions more difficult to name than those of the partitioned stimuli, as evidenced by longer RTs and more frequent errors. The difference between partitioned and unitary stimuli was much reduced for the second graders, who were averaging 12 names in about $10 \mathrm{sec}$. The significant interaction between type of stimuli and grade for RTs reflects this developmental change in the difference in ease of accessing the two types of stimuli. Although the interaction was not significant in the error data, the trend was the same.

Qualitatively, it was apparent that the children, and particularly the younger children, found the naming of the dimensional values to be difficult, especially for the unitary stimuli. Although the total time spent in the experimental situation for each child was considerably less than that in many learning experiments that we have conducted, the kindergarten and first-grade children gave considerable indication that the task was demanding. This observation is consistent with one we made in an earlier

Table 1

Mean Response Time (in Seconds) and Errors for Kindergarten, First-Grade, and Second-Grade Children During the Naming of Dimensional Values of Partitioned and Unitary Stimuli

\begin{tabular}{|c|c|c|c|c|c|c|}
\hline \multirow[b]{2}{*}{ Grade } & \multicolumn{3}{|c|}{ Mean Response Time } & \multicolumn{3}{|c|}{ Mean Errors } \\
\hline & Partition & Unitary & Combined & Partition & Unitary & Combined \\
\hline Kindergarten & 14.72 & 17.01 & 15.86 & 0.39 & 0.49 & 0.44 \\
\hline First & 11.96 & 15.00 & 13.48 & 0.22 & 0.52 & 0.37 \\
\hline Second & 9.60 & 10.46 & 10.03 & 0.16 & 0.15 & 0.16 \\
\hline Combined & 12.09 & 14.16 & & 0.26 & 0.39 & \\
\hline
\end{tabular}


experiment (Spiker \& Cantor, 1980) in which kindergarten children were asked to name the dimensional values of unitary stimuli.

It seems reasonable to conclude that the speed with which children can name the dimensional values of multidimensional stimuli is a valid indication of the ease with which they can access the dimensional structure of such stimuli. Thus, the method developed in this study shows considerable promise as a means for studying the continuum between stimulus dimensions that are completely integral and those that are separable, but not easily accessed, by the young child.

\section{REFERENCES}

Cantor, J. H., \& Spiker, C. C. The problem-solving strategies of kindergarten and first-grade children during discrimination learning. Journal of Experimental Child Psychology, 1978, 26, 341-358.

Garner, W. R. The stimulus in information processing. American Psychologist, 1970, 25, 350-358.

GARNER, W. R. The processing of information and structure. Hillsdale, N.J: Erlbaum, 1974.

GaRner, W. R., \& Felfoldy, G. L. Integrality of stimulus dimensions in various types of information processing. Cognitive Psychology, 1970, 1, 225-241.

KemLer, D. G. The ability for dimensional analysis in preschool and retarded children: Evidence from comparison, conservation and prediction tasks. Journal of Experimental Child Psychology, 1982, 34, 469-489.

KEMLER, D. G. Holistic and analytic modes in perceptual and cognitive development. In T. J. Tighe \& B. E. Shepp (Eds.), Perception, cognition, and development: Interactional analyses. Hillsdale, N.J: Erlbaum, 1983.
KemLer, D. G., \& Sмith, L. B. Accessing similarity and dimensional relations: The effects of integrality and separability on the discovery of complex concepts. Journal of Experimental Psychology: General, 1979, 108, 133-150.

LOCKHEAD, G. R. Effects of dimensional redundancy on visual discrimination. Journal of Experimental Psychology, 1966, 72, 95-104.

ShePARD, R. N. Attention and the metric structure of the stimulus space. Journal of Mathematical Psychology, 1964, 1, 54-87.

SHE PP, B. E. The analyzability of multidimensional objects: Some constraints on perceived structure, the development of perceived structure, and attention. In T. J. Tighe \& B. E. Shepp (Eds.), Perception, cognition, and development: Interactional analyses. Hillsdale, N.J: Erlbaum, 1983.

She PP, B. E., \& Swartz, K. B. Selective attention and the processing of integral and non-integral dimensions: A developmental study. Journal of Experimental Child Psychology, 1976, 22, 73-85.

Smith, L. B. Perceptual development and category generalization. Child Development, 1979, 50, 705-715.

Smith, L. B., \& Kemler, D. G. Developmental trends in free classification: Evidence for a new conceptualization of perceptual development. Journal of Experimental Child Psychology, 1977, 24, 279-298.

Spiker, C. C., \& Cantor, J. H. The effects of stimulus type, training, and chronological age on children's identification and recoding of multidimensional stimuli. Journal of Experimental Child Psychology, 1980, 30, 144-158.

Spike r, C. C., \& Cantor, J. H. Components in the hypothesistesting strategies of young children. In T. J. Tighe \& B. E. Shepp (Eds.), Perception, cognition, and development: Interactional analyses. Hillsdale, N.J: Erlbaum, 1983.

(Manuscript received for publication August 1, 1983.) 